\title{
A pandemia de SARS-CoV-2: Cenários epidemiológicos e medidas de correlações
}

\section{The SARS-CoV-2 pandemic: epidemiological scenarios and correlation measures}

Jefferson Oliveira do Nascimento ${ }^{1}$, Carlos Augusto Cardoso Passos ${ }^{2}$, Joana D'Arc Silva Galvão de Carvalho ${ }^{3}$, Estéfano Aparecido Vieira ${ }^{4}$, Marcelo Albano Moret $^{5}$.

\section{RESUMO}

A pandemia de Coronavírus se apresentou como um dos problemas mais complexos de saúde pública, em sua magnitude, até o presente momento da história da humanidade. Uma possibilidade de orientar as políticas públicas para combater esta enfermidade é a proposição de modelos preditivos em relação a difusão do SARSCoV-2. Este artigo tem como objetivo apresentar alguns modelos epidemiológicos, como o modelo SEIAHR e métodos de ajustes não-lineares (por meio de Gaussianas) utilizados para realizar estimativas sobre a propagação do Coronavírus em países como a Itália e Brasil. Destaca-se a proposição de um Modelo de Física do Estado Sólido como método de previsão. Uma curva sigmoide especificamente utilizada em processos de transformação de materiais sólidos: a equação de Avrami ou de Johnson-Mehl-Avrami-Kolmogorov (JMAK). Também é apresentado em que nível um Estado da Região Norte (Pará) e um do Sudeste (Espírito Santo) apresentaram um comportamento de correlação na difusão do Coronavírus em território nacional, em comparação com os demais Estados Brasileiros. O percurso metodológico é por meio de métricas de correlações estatísticas. Ao fim, podemos inferir que as metodologias utilizadas foram capazes de propor um padrão quanto ao processo de disseminação do Coronavírus acerca das regiões estudadas.

Palavras-chave: Palavras-chave: Cenários epidemiológicos, Coronavírus, Covid-19

\section{ABSTRACT}

The Coronavirus pandemic has been one of the most complex public health problems, in its magnitude, up to the present moment in human history. One possibility to guide public policies to combat this disease is the proposition of predictive models regarding the dissemination of SARS-CoV-2. This article aims to present some methods, nonlinear fits and computational models used to estimate Coronavirus propagation in countries such as Italy and Brazil. We highlight the proposition of a Solid State Physics Model as a forecasting method. A sigmoid curve specifically used in solid materials transformation processes: the Avrami or Johnson-Mehl-Avrami-Kolmogorov (JMAK) equation. Also noteworthy are the SEIAHR model and the non-linear adjustments through Gaussians. Finally, we can infer that the methodologies used were able to propose a pattern for the process of dissemination of Coronavirus in the regions studied.

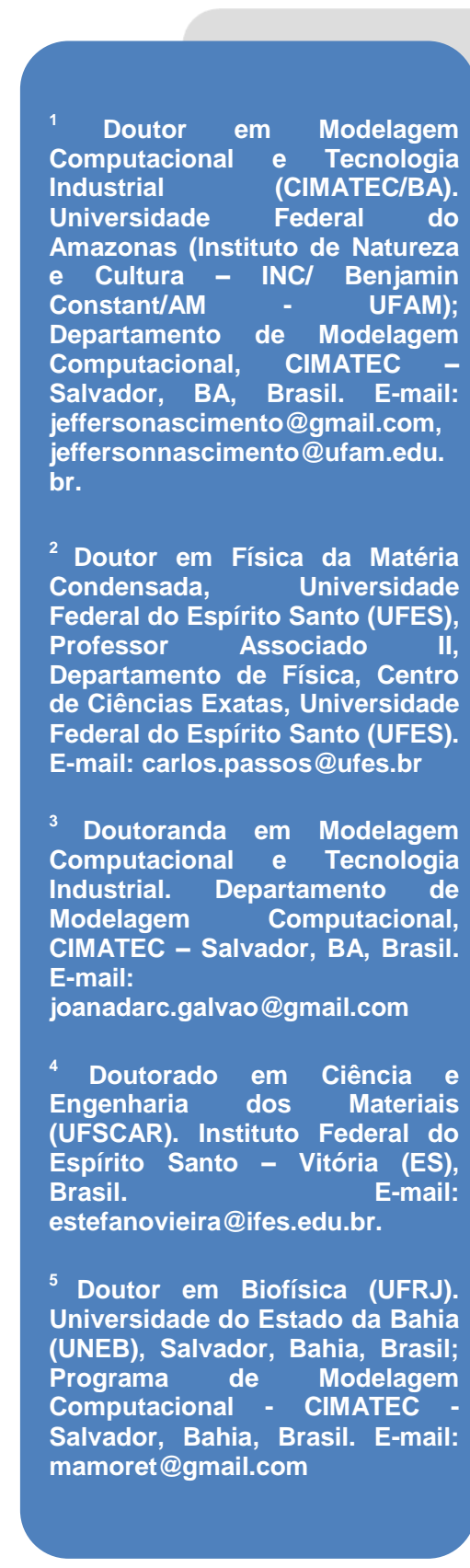

Keywords: Epidemiological scenarios, Coronavirus, Covid-19 


\section{INTRODUÇÄO}

A pandemia causada pelo vírus SARS-CoV-2, agente etiológico da Covid-19 (i.e. Conronavirus disease), representa uma ameaça de magnitude não enfrentada neste século. Por causa disto, cada governo propôs medidas de saúde pública fundamentais para se retardar a transmissão e a propagação do vírus e mitigar seus impactos. Especificamente o surto no Brasil, desencadeou um grande conjunto de casos infectados por Covid-19.

Durante quase todo ano de 2020, além não haver medicamentos ou vacinas comprovadamente científicas para o tratamento da doença, foi e ainda é muito importante fazer análises dos dados de novos casos e óbitos e a sua interpretação matemática para fundamentar as medidas governamentais para a supressão e mitigação do impacto da Covid-19. Isto significa que é/foi necessário fazer estimativas através de modelos matemáticos para avaliar o potencial de desenvolvimento de transmissão humanohumano sustentado.

Uma vez que a doença apresenta suas próprias características biológicas, os modelos precisam ser adaptados à variabilidade das nuances das regiões, das tomadas de decisões governamentais e da população, a fim de serem capazes de lidar com situações reais. Assim, neste artigo, temos a intenção de colaborar com a compreensão do quadro epidemiológico em 2020 e 2021 através do estudo de um modelo matemático por equações diferenciais ordinárias e modelo similares que possam descrever a propagação da doença infecciosa de transmissão pessoa a pessoa.

Nosso intuito de seguir neste viés, ocorre pela necessidade em estimativas em relação a pandemia de Covid-19, pois, os números reais dependem totalmente da testagem em massa da população (NASCIMENTO, 2020). Algo que não ocorre (até este momento) no Brasil e, em muitos países. Por exemplo, em estudos recentes sobre a pandemia surgem trabalhos científicos que sugerem para o Estado do Pará a multiplicação dos números oficiais de novos casos por valores entre 7-20 (ibidem).

Diante do exposto, o objetivo da presente pesquisa é apresentar cenários epidemiológicos à difusão do Coronavírus. Utilizaremos alguns métodos tradicionais em Epidemiologia por meio da modelagem computacional, ajustes não-lineares e o Modelo da equação de Avrami ou de Johnson-Mehl-Avrami-Kolmogorov (JMAK), que apresentou resultados robustos para tal propagação viral. 


\section{MATERIAIS E METODOS}

Para que pudéssemos realizar as análises propostas neste artigo, a base de dados oficial utilizada é a fornecida pelo Ministério da Saúde. Para realizarmos análises preditivas em relação à difusão do Coronavírus, apresentaremos alguns métodos que utilizaremos nesta pesquisa.

\subsection{Modelo SIR}

Este modelo epidemiológico foi proposto por Kermack e McKendrick (1927), leva em consideração as seguintes variáveis:

1. Indivíduos susceptíveis que ainda não foram contaminados pela doença. $\mathrm{S}(\mathrm{t})$ é usado para representar o número de indivíduos não infectados com a doença no momento $\mathrm{t}$, ou aqueles suscetíveis à doença.

2. Indivíduos infectados que são aqueles que tiveram contato com a doença (contaminados). I(t) representa o número de indivíduos que tenham sido infectadas com a doença e que são capazes de transmitir a doença aos da categoria susceptível.

3. Indivíduos removidos que são representados por aqueles recuperados (curados) e mortos. $\mathrm{R}(\mathrm{t})$ é o compartimento utilizado para aqueles indivíduos que foram infectados e, em seguida, removidos a partir da doença, quer devido à imunização ou devido à morte. Vamos considerar que nesta categoria as pessoas curadas não são capazes de serem infectados novamente ou para transmitir (numa janela de tempo) a infecção a outras pessoas. O fluxo do presente modelo pode ser considerado da seguinte forma:

$$
S \rightarrow I \rightarrow R
$$

Levamos em consideração que o número de indivíduos em cada uma das três classes muda em relação ao tempo, isto é, $S(t), I(t)$ e $R(t)$ são funções dependentes do tempo $t$. Portanto, o total da população é a soma do número de indivíduos que estão em cada uma das classes em qualquer tempo $t: \mathrm{N}=S(t)+I(t)+R(t)$. Isto simplifica o modelo. 
Kermack e McKendrick (1927) apresentam um modelo matemático cuja distribuição estatística segue uma normal, que denota os casos que são removidos por morte ou recuperação em uma epidemia:

$$
\frac{d z}{d t}=\frac{l^{3}}{2 x_{0} k^{2}} \sqrt{-q} \sec h^{2}\left(\frac{\sqrt{-q}}{2} l t-\phi\right)
$$

Conforme Savi, Savi e Borges (2020) o modelo SIR busca resolver o sistema de equações diferenciais ordinárias: $\frac{d S}{d t}=-\beta \frac{I}{N} S, \frac{d I}{d t}=\beta \frac{I}{N} S-\gamma I$ e $\frac{d R}{d t}=\gamma I$. Tais equações podem ser simplificadas da seguinte maneira:

$$
\begin{aligned}
& \frac{d S}{d t}=-\lambda S \\
& \frac{d I}{d t}=\lambda S-\gamma I \\
& \frac{d R}{d t}=\gamma I
\end{aligned}
$$

Nas equações anteriores $\lambda$ é a taxa na qual os indivíduos estão suscetíveis a ficarem infectados (i.e. "a força da infecção") (SAVI; SAVI; BORGES, 2020) e $\gamma$ é a taxa de recuperação para indivíduos infectados. Ainda Devemos considerar também as seguintes hipóteses sobre o modelo:

- todos os indivíduos nascem suscetíveis;

- infectados que se recuperam, ganham imunidade total;

- as interações entre os componentes se dão de forma homogênea;

- o tamanho da população permanece constante em relação ao tempo;

- não consideramos emigração ou imigração;

- não consideramos vacinação (campanha de imunização).

$\mathrm{Na}$ literatura científica há variações deste modelo quando se considera outros fatores, como apresentado na próxima subseção. 


\subsection{Modelo SEIRD}

No modelo SEIRD, dividimos a população em 5 grupos: suscetível, exposto, infectado, recuperado e morto. Os parâmetros são similares daqueles do modelo SRI. A população exposta difere da população infectada sobre o desenvolvimento de sintomas; um indivíduo com o vírus entra primeiro no grupo exposto, carregando o vírus durante o período de incubação; então, com o desenvolvimento dos sintomas, o indivíduo passa para o grupo infectado. $O$ fluxo do presente modelo pode ser considerado da seguinte forma:

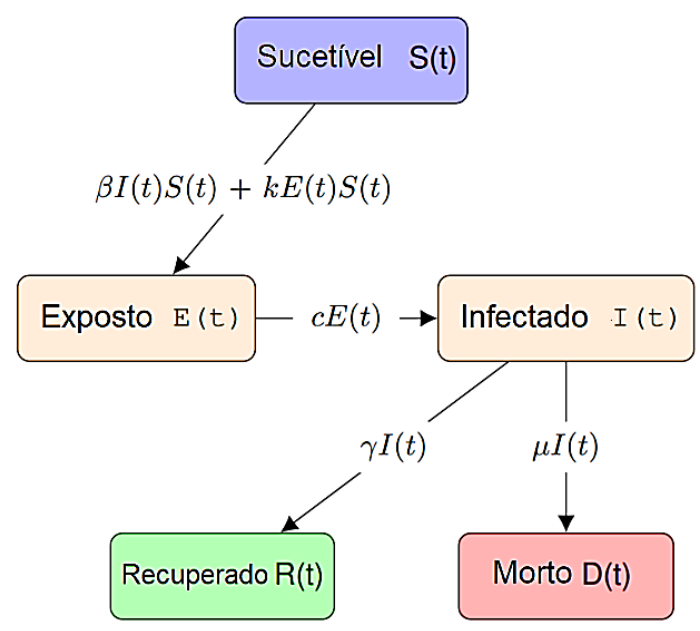

Figura 1. Modelo SEIRD. Fonte: Cintra e Fontinele (2020).

A equações diferenciais que descrevem o modelo são:

$$
\begin{aligned}
& \frac{d S}{d t}=-\frac{\beta}{N} I(t) S(t)-\frac{k}{N} E(t) S(t) \\
& \frac{d E}{d t}=\frac{\beta}{N} I(t) S(t)+\frac{k}{N} E(t) S(t)-c E(t) \\
& \frac{d I}{d t}=c E(t)-\gamma I(t)-\mu I(t) \\
& \frac{d R}{d t}=\gamma I(t) \\
& \frac{d D}{d t}=\mu I(t)
\end{aligned}
$$


A taxa de recuperação é representada por $\gamma$ e a taxa de mortalidade $\mu$ são representadas em termos da taxa de mortalidade por casos (CFR) $P$ :( e o tempo médio desde o início dos sintomas até a recuperação $\tau_{r}$ e morte $\tau_{d}$.

$$
\begin{gathered}
\mu=\frac{P_{:(}}{\tau_{d}} \\
\gamma=\frac{1-P_{:(}}{\tau_{r}}
\end{gathered}
$$

Todas as equações consideram que a população total $N$ se conserva no tempo e é homogênea por simplicidade (CINTRA; FONTINELE, 2020).

\subsection{Modelo de Física do Estado Sólido como Modelo de Previsão}

Uma das curvas mais conhecida é a curva "sigmóide" amplamente utilizada em Ciências e Engenharia de Materiais, Computação, Economia e Sistemas Ecológicos. $\mathrm{O}$ nome "sigmóide" vem da forma em $\mathrm{S}$ do seu gráfico. Especificamente, em processos de transformação de materiais sólidos, temos a equação de Avrami, algumas vezes chamada de equação de Johnson-Mehl-Avrami-Kolmogorov (JMAK) (PASSOS et al., 2020).

A expressão de JMAK estabelece que a cristalinidade desenvolvida por um material (cerâmica, fármacos, metais, polímeros) (SHI et al., 2020; BRITO et al., 2012) aquecido em regime isotérmico, por um tempo $t$, pode ser correlacionada com o tipo e a cinética de nucleação e crescimento cristalino. Assim, quando as suposições da teoria JMAK são satisfeitas, isto é, quando amostra é infinitamente grande, a nucleação espacialmente é randômica e as taxas de nucleação e crescimento são independentes do tempo, temos

$$
f(t)=1-\exp \left(-k t^{n}\right)
$$

onde $f(t)$ é a fração de massa cristalizada no tempo $t$, $k$ é a constante cinética e $n$ é o expoente de Avrami, real e positivo. Para obtenção dos parâmetros $n$ e $k$, devemos linearizar a Eq. (1) como se segue: $1-f(t)=\exp \left(-k t^{n}\right)$, passando a ser, $-\ln [1-f(t)]=\left(-k t^{n}\right)$ e, na sequência: 


$$
\ln \{-\ln [1-f(t)]\}=n \ln (t)+\ln (k)
$$

Verificamos que esta dinâmica de transformação de fase para materiais se assemelha a dinâmica de uma epidemia (e neste caso, a pandemia por Coronavírus) PASSOS et al., 2020). Assim, vamos assumir que o parâmetro $k$ representa a taxa de nucleação e de crescimento de casos. $O$ tempo $t=0$ corresponde à primeira confirmação da doença pelas autoridades de saúde, e, portanto, considerado como o início da epidemia. Para tal análise, devemos construir um gráfico com número de casos que evoluíram no tempo $\ln \{-\ln (1-f(t))\}$ versus $\ln (t)$. Para esta figura comparamos a eq. (2) com uma função linear:

$$
\begin{aligned}
\ln \{-\ln [1-f(t)]\} & =n \ln (t)+\ln (k) \\
y & =a x+b
\end{aligned}
$$

A Eq. 13, corresponde a uma função do tipo $y=a x+b$, em que a $=n$ e $b=\ln (k)$. Em seguida, usamos estes parâmetros de ajustes para encontrar o número de casos totais e número diário de casos. A Equação 14 é a expressão original da teoria JMAK:

$$
f(t)=1-\exp \left(-\frac{4}{3} \pi I G^{3} t^{n}\right)
$$

em que:

$I$ = o número de núcleos ou uma taxa de emergência de núcleos;

$\mathrm{G}=\mathrm{a}$ taxa de crescimento de cada núcleo;

$t=$ tempo, nosso período de análise;

$n=$ um parâmetro que representa se o sistema segue a saturação do site. Dessa forma, o valor será igual a 3; sob taxa de nucleação constante, o valor será igual a 4.

No caso de taxa de nucleação constante, a taxa de emergência de núcleos é descrita pela Equação 15:

$$
I=I_{0} e\left(-\frac{Q}{R T}\right)
$$

em que: 
$Q=$ energia de ativação;

$R=$ constante de gás ideal ( $R=8,314 \mathrm{~J} / \mathrm{K} \cdot \mathrm{mol})$.

Quando a Equação 14 é aplicada a processos de transformação de fase de materiais, a taxa de nucleação constante deve ser considerada em duas situações diferentes. O primeiro é com a saturação do sítio, em que os núcleos aparecem por um determinado tempo $\mathrm{t}=0$ e todos crescem na mesma taxa. Assim, o volume de cada núcleo é o mesmo para todos os núcleos até que ocorra 100\% da transformação. Com o tempo, nenhum novo núcleo aparece, e o termo I na Eq. 14 assume um valor fixo em todo o processo de transformação (por exemplo, cinco mil núcleos). Na segunda situação, não há saturação de sítio e os núcleos aparecem à medida que os mais velhos crescem, então lé uma taxa (por exemplo, cinco mil núcleos / s).

Quando generalizamos a Eq. 14, obtemos a supracitada Eq. 12. Destacamos que desta última equação, $k$ corresponde à seguinte expressão matemática:

$$
k=k_{0} \exp \left(-\frac{Q}{k_{b} T}\right)
$$

Em um processo de transformação de fase, representa a energia de ativação, $k_{B}$ é a constante de Boltzman e $T$ é a temperatura. Em casos práticos, os valores de $k$ e $n$ podem assumir valores diferentes, considerando que os núcleos não são perfeitamente esféricos, a taxa de nucleação não é constante, entre outras variáveis práticas não previstas.

\subsubsection{Descrição dos casos de COVID-19 com o modelo Johnson- Mehl - Avrami - Kolmogorov}

Nesta pesquisa, as pessoas não contaminadas serão o universo a ser transformado do estado não contaminado para o contaminado. Assim, para a aplicação da equação JMAK em casos de epidemias, consideramos que:

- a temperatura $T$ representa o grau de pessoas circulando nas ruas, em seus bairros, e fazendo a pandemia crescer na fronteira contaminada, sem o transporte de longa distância para formar novos núcleos;

- o parâmetro $k$ descreve a taxa de expansão dos casos COVID-19 ao longo do tempo - consideramos k uma constante na primeira aproximação, uma vez que $T$ é constante (representa 0 número de pessoas contaminadas avançando 
na vizinhança não contaminada a uma taxa constante);

- $\quad f$ é a fração de pessoas infectadas em uma população;

- $\quad n$ representa a capacidade de criação de novos núcleos de pessoas contaminadas e está associado ao transporte de pessoas em meios de transporte de longa distância, como avião, metrô, ônibus e trem.

Ressaltamos que o número de pessoas imunizadas e que vão a óbito não foi considerado. Em nosso modelo, uma pessoa infectada com a COVID-19, quando em uma região não afetada pela doença, pode gerar um núcleo (cluster 1) de pessoas infectadas em um determinado momento, que consideramos como o momento inicial $(t=0)$ do surto da doença. Nessa situação, algumas pessoas adquirem a doença. Em um momento, uma pessoa desse núcleo se desloca para outra região onde não há infecção por algum meio de transporte público, podendo surgir um segundo núcleo (cluster 2).

Consideramos que os clusters possuem tamanhos diferentes, pois foram criados em momentos distintos e o núcleo contaminado cresce com o tempo, conforme mostrado nas Figuras $2 \mathrm{~A}$ e $2 \mathrm{~B}$. Se nenhuma medida for tomada para conter o surto da doença, outras pessoas infectadas criam núcleos em regiões ainda não afetadas pelo vírus. Em um dado momento $t_{x}>0$, teremos outros núcleos formados, como mostra a Figura 2C.

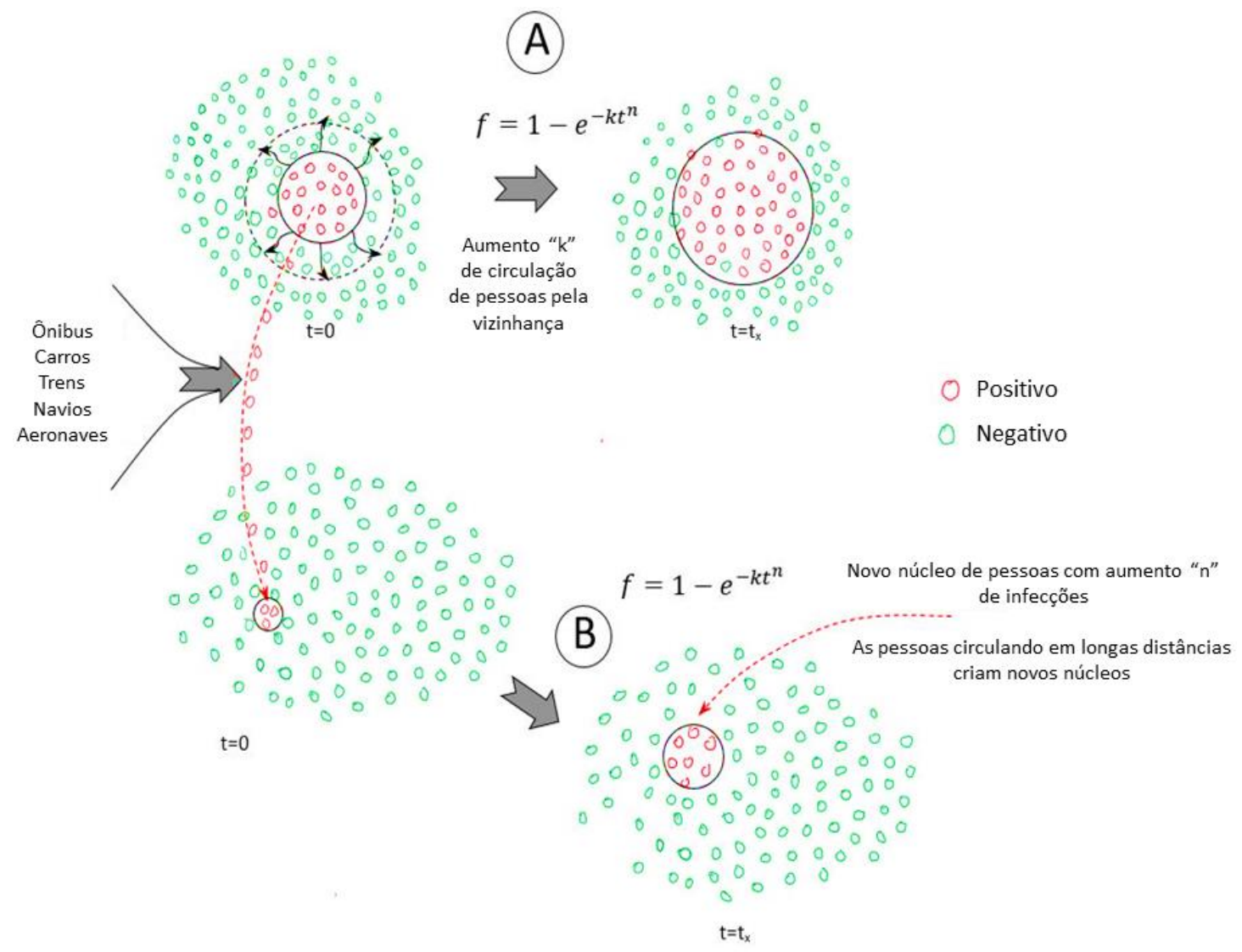




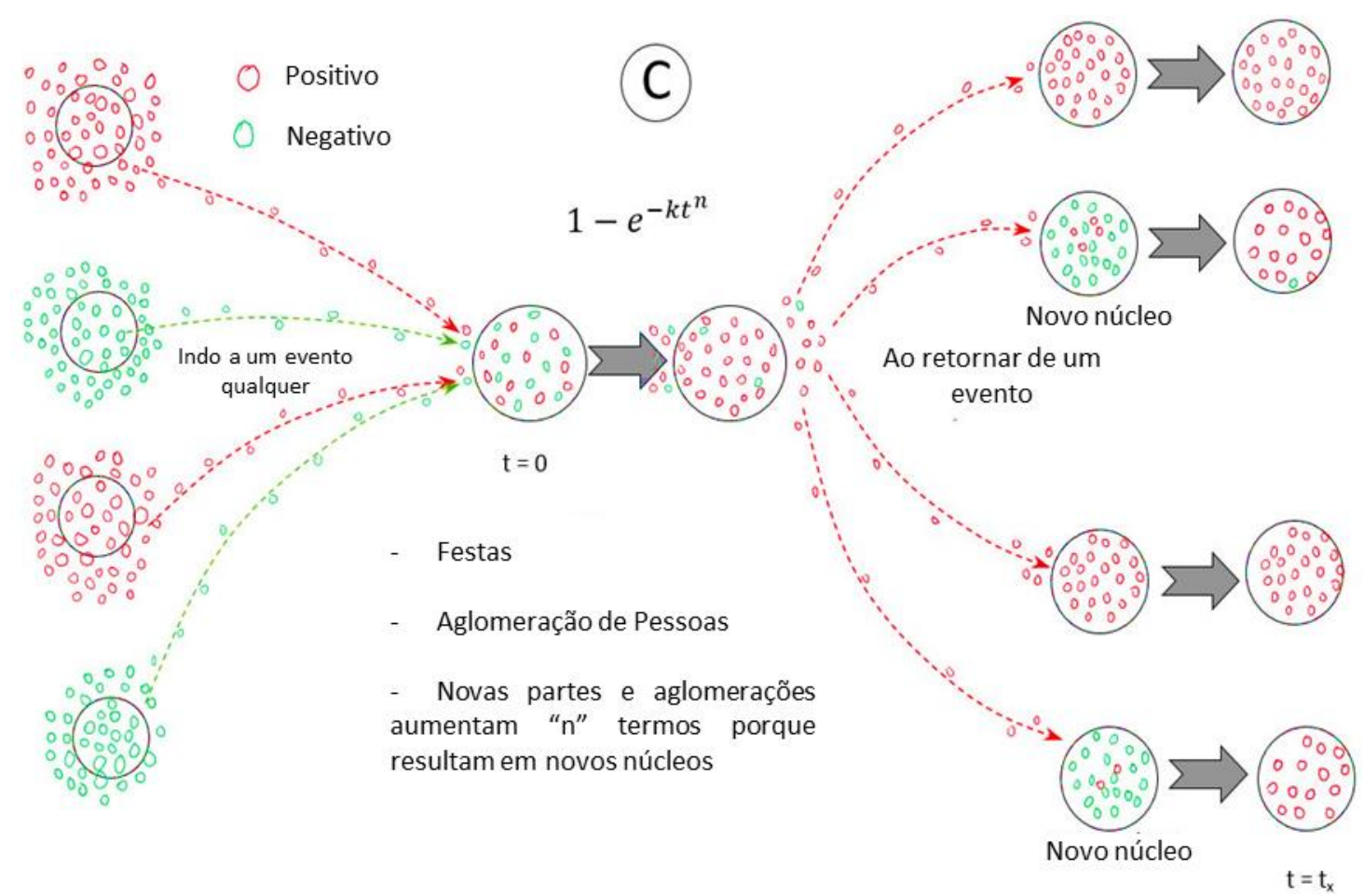

Figura 2. Modelo de difusão do novo Coronavírus. Figuras 2A, 2B e 2C: formação de novos núcleos de infecções por meio de atividades que aglomeram pessoas. Fonte: Dos autores.

\subsection{Modelo SEIAHR}

O Método SEIAHR, que tem como significado Susceptible, Exposed, Infected, Asymptomatic, Hospitalized, Rehabilitated, em português: Suscetíveis, Expostos, Infectados, Assintomáticos, hospitalizados e Reabilitados e, é responsável por justamente realizar uma modelagem da evolução temporal entre estas variáveis. Com as considerações realizadas em Rocha Filho et al. (2020) passamos a ter neste modelo epidemiológico para o SARS-CoV-2: Indivíduos Susceptíveis $\left(\mathrm{S}_{\mathrm{i}}\right)$, Hospitalizados $\left(\mathrm{H}_{\mathrm{i}}\right)$ por Covid-19, Expostos $\left(E_{i}\right)$ (indivíduos em período de incubação viral e que não estão transmitindo a infecção), Infecciosos $\left(\mathrm{l}_{\mathrm{i}}\right)$ e Recuperados $\left(\mathrm{R}_{\mathrm{i}}\right), i=1, \ldots, \mathrm{M}$. Para $\circ t=0$, temos $\sum_{i=1}^{M} n_{i}=1$; em que a população é representada por um determinado grupo de faixa etária de idade $n_{i}=S_{i}+H_{i}+E_{i}+I_{i}+R_{i}$ e variável $\mathrm{M}$, corresponde ao número de grupos por faixa etária de idade (0-9, 10-39, 40-49, 50-59, 60-69, $\geq 70$ anos).

A probabilidade de transmissão é então obtida realizando alguns ajustes, levando em conta, de forma básica a quantidade do número de interações pessoais $R_{0}=\sum_{i, j=1}^{M} n_{j} \beta_{i j} / \gamma$, ou: 


$$
R_{0}=\sum_{i, j=1}^{M} n_{j} P_{c} C_{i j} / \gamma
$$

É suposto também, para a Eq. 17, que somente os casos graves e críticos sejam hospitalizados (ROCHA FILHO et al., 2020). No viés de que os enfermos que se encontram hospitalizados estão na condição de não contato com as pessoas externas ao ambiente hospitalar, obviamente, eles não contribuirão para a força de infecção, obtida da seguinte forma:

$$
\lambda_{i}=\sum_{i=1}^{M} B_{i, j} \frac{I_{j}}{n_{i}}
$$

Assim, o sistema de equações diferenciais que representam o modelo são as seguintes:

$$
\begin{aligned}
& \frac{d S_{i}}{d t}=\kappa \delta_{i, 1}-\lambda_{i} S_{i}-\mu S_{i}+v_{i} S_{i}+v_{i-1} S_{i-1} \\
& \frac{d H_{i}}{d t}=-\psi H_{i}+\zeta_{i} \lambda_{i} E_{i}\left(t-\tau_{1}\right)-\theta \zeta_{i} \lambda_{i} E_{i}\left(t-\tau_{2}\right)-\mu H_{i} \\
& \frac{d E_{i}}{d t}=\lambda S_{i}-\sigma E_{i}-\zeta_{i} \sigma E_{i}-\mu E_{i}-v_{i} E_{i}+v_{i-1} E_{i-1} \\
& \frac{d I_{i}}{d t}=\sigma E_{i}-\gamma I_{i}-\mu I_{i}-v_{i} I_{i}+v_{i-1} I_{i-1} \\
& \frac{d R_{i}}{d t}=\gamma I_{i}-\psi H_{i}-\mu R_{i}-v_{i} R_{i}+v_{i-1} R_{i-1}
\end{aligned}
$$

Para todas as equações diferenciais apresentadas, a dependência das variáveis é em função do tempo, com exceção a Eq. 19, com a presença do delta de Kronecker $\left(\delta_{i, 1}\right)$, em que assume o valor 1 (se $i=j$ ) e, caso ao contrário, o valor será 0) ROCHA FILHO et al., 2020). Apresentamos na Figura 3, o modelo esquemático para a Eq. 23, cuja taxa de transmissão viral é dada pela Eq. (17) e a força de infecção é a obtida pela Eq. (18): 


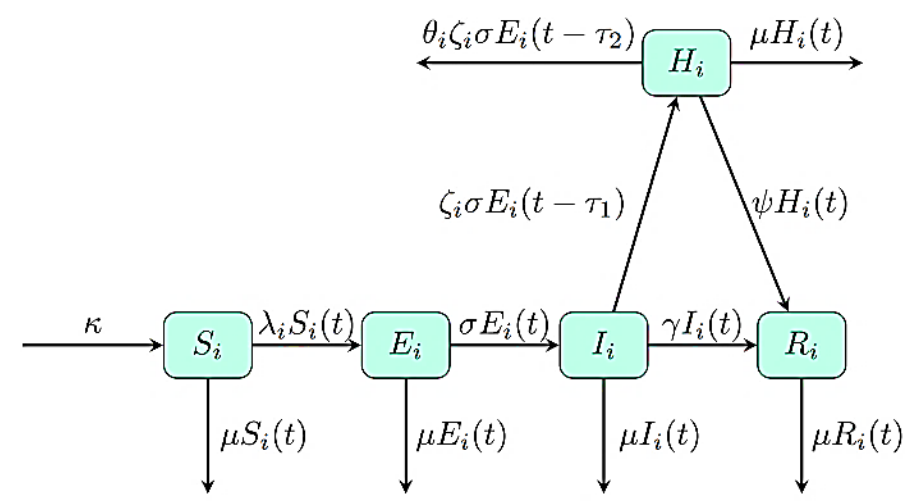

Figura 3: Modelo SEIAHR. Fonte: Adaptado de Rocha Filho [8].

\subsection{Modelos preditivos baseados em Gaussianas}

A distribuição de probabilidade normal ou Gaussiana de uma variável $x$, que segue uma distribuição de probabilidades chamada desta forma, tem sua função densidade de probabilidades obtida da seguinte maneira:

$$
f_{x}(x)=\frac{1}{\sqrt{2 \pi} \sigma_{x}} \exp \left[-\frac{1}{2}\left(\frac{x-\mu_{x}}{\sigma_{x}}\right)^{2}\right]
$$

A Eq. 24 depende da média e do desvio padrão da variável $x$, ou seja, $N\left(\mu_{x}, \sigma_{x}\right)$ (IBARRA (2017).A distribuição Normal Padrão, é dada pela seguinte relação :

$$
f_{y}(y)=\frac{1}{\sqrt{2 \pi}} \exp \left[-\frac{1}{2} y^{2}\right]
$$

Além das Eq. 24 e Eq. 25, destacamos que a Estatística de Ordem possibilita encontrarmos da distribuição de valores extremos de interesse por meio de uma distribuição inicial da variável aleatória, considerando hipóteses que possam simplificar o modelo [10]. Assim, destacamos as formas assintóticas de convergência, que dependem do decaimento da calda de distribuição inicial, na direção do extremo de interesse. Neste viés, as distribuições estatísticas mais utilizadas são a de Gumbel, Fréchet ou de Weibull (ibidem).

O comportamento realizado pelo Coronavírus, em boa medida, assim como nos modelos preditivos apresentados anteriormente, também pode ser aproximado, considerando um ajuste não-linear por meio do exposto nesta sessão, das Gaussianas. 


\subsection{Medidas de correlações estatísticas}

Diante do exposto nas sessões anteriores, este artigo também tem como objetivo investigar em que medida há correlações estatísticas, especificamente, entre o Estado do Espírito Santo e do Pará com os demais Estados Brasileiros e o Distrito Federal, especificamente em relação às curvas que representam o número de novos casos de infecções diários e novos casos de óbitos diários. Destacamos que o espaço amostral utilizado corresponde desde o primeiro caso oficial registrado no Brasil, que ocorreu na quarta semana de fevereiro de 2020 até trinta de novembro de 2020.

Para que pudéssemos realizar as medidas de correlações, construímos para os 26 Estados e mais o DF, uma representação matemática por meio de séries temporais. Desta forma, estabelecemos as comparações que nos interessavam, para o Pará e o Espírito Santo, em relação ao número diário de infecções e óbitos. Calculamos primeiramente a correlação por meio do coeficiente de correlação de Pearson (r). Para tal, o nível de correlação entre as séries temporais construídas, utilizamos primeiramente o coeficiente de Pearson, proposto por Karl Pearson (1857-1936), conforme Eq. 26:

$$
r=\frac{n \sum x_{i} y_{i}-\left(\sum x_{i}\right) \cdot\left(\sum y_{i}\right)}{\sqrt{\left[n \sum x_{i}^{2}-\left(\sum x_{i}\right)^{2}\right] \cdot\left[n \sum y_{i}{ }^{2}-\left(\sum y_{i}\right)^{2}\right]}}
$$

De forma simplificada, destacamos que, quando $r=+1$ a correlação é perfeitamente positiva. Se $r=-1$ a correlação é perfeitamente negativa e se $r=0$ não há correlação entre os pontos ou há, porém, é não-linear (NASCIMENTO, 2019; NASCIMENTO, 2020). Em relação ao coeficiente de Spearman $\left(r_{s}\right)$, baseada em postos, é o índice de correlação mais antigo a ser utilizado. Ele exige que as variáveis, em caso de correlação X e Y, tenham suas métricas por meio de escala ordinal (BAUER, 2007). Caso $\mathrm{X}$ e $\mathrm{Y}$ sejam quantitativas e sua distribuição de graus seja não bivariada, Spearman é uma alternativa em substituição a Pearson. Caso correlação de Spearman, ao utilizar os dados do espaço amostral, sejam substituídos por postos ordenados, poderá ser calculada de forma semelhante ao coeficiente de Pearson. A principal vantagem do coeficiente de correlação de Spearman $\left(r_{s}\right)$ é a não exigência da normalidade dos dados, da seguinte forma: 


$$
r_{s}=1-\frac{6 \cdot \sum_{i=1}^{n} d_{i}^{2}}{n^{3}-n}
$$

Em que $d_{i}=r_{X i}-r_{Y i}$, variam de 1 a $n$. (CONOVER, 1998).

A Eq. 27 corresponde ao coeficiente de correlação para postos de Spearman $\left(r_{s}\right)$. Destacamos algumas características: Não há problema em sua utilização mesmo com à presença de outliers. -1 indica perfeita correlação negativa ou inversa e 1 indica perfeita correlação positiva ou direta.

Em relação ao coeficiente de correlação por postos de Kendall $(\tau)$, corresponde a uma medida de associação, também para variáveis ordinais. Porém, este coeficiente de correlação indicará em que medida há um grau de associação entre dois conjuntos de postos (MIOT, 2018). O Nível da medida do relacionamento entre dois conjuntos de postos é obtido pela razão entre o total efetivo de escores +1 e -1 , em relação ao total máximo possível.

Considerando o número de pares concordantes e discordantes, em relação ao número total de pares do espaço amostral (desconsiderando os valores repetidos), encontraremos coeficiente de correlação por postos de Kendall $(\tau)$ (SANTOS, 2012). De outra forma: Considerando o total possibilidades por $S$ e o quantitativo máximo de escores possíveis por $\left(\begin{array}{l}n \\ 2\end{array}\right)=n \frac{(n-1)}{2}$, o coeficiente de correlação de Kendall da seguinte maneira:

$$
\begin{gathered}
\tau=\frac{S}{n \frac{(n-1)}{2}} \\
\tau=\frac{2 S}{n(n-1)}
\end{gathered}
$$

Em relação aos outliers, o coeficiente de correlação por postos de Kendall $(\tau)$ é uma métrica estatística robusta em caso de termos no espaço amostral, com a presença de dados extremos (MIOT, 2018). 


\section{RESULTADOS E DISCUSSAOO}

\subsection{Cenários epidemiológicos}

Por meio das principais metodologias dispostas na sessão anterior, apresentamos na sequência, alguns resultados já estimados em relação à Pandemia de Coronavírus. Nas Figura 4, apresentamos algumas estimativas para a Itália:

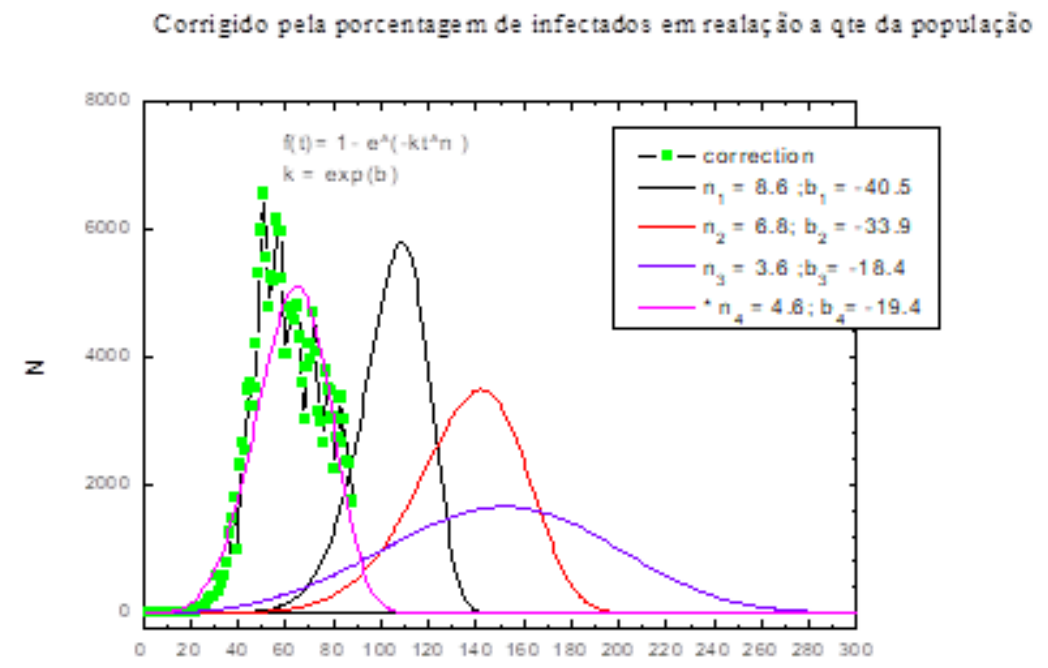

Figura 4: Estimativa realizada por meio da equação de Avrami (JMAK), para a Itália (realizado em 27/04/2020). Fonte: Dos autores.

$\mathrm{Na}$ Figura 5, algumas estimativas realizadas para o Brasil, observando os dados fornecidos pelo Ministério da Saúde, até o final de Maio/2020:

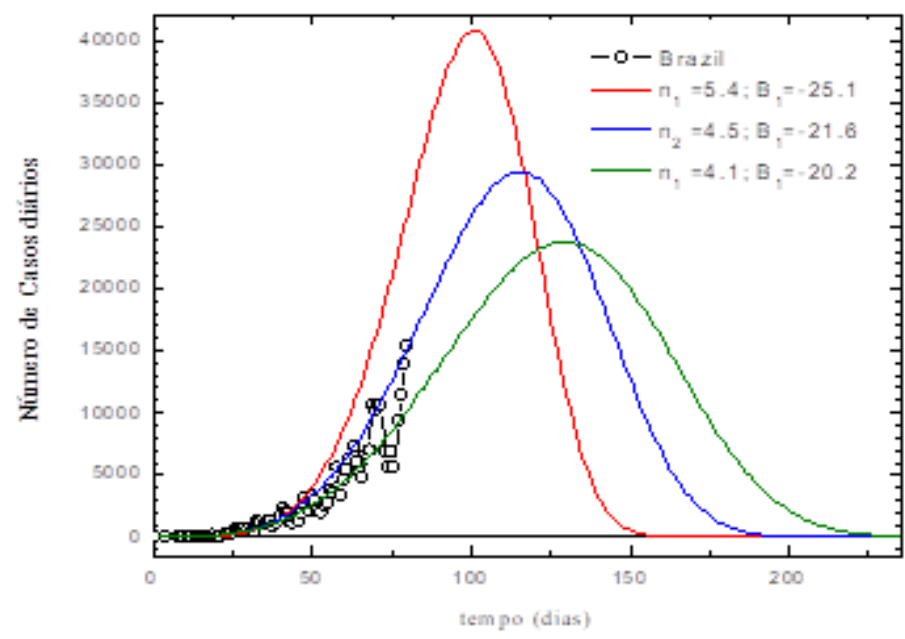

Figura 5. Estimativas realizadas por meio da equação de Avrami (JMAK), para o Brasil (realizado final de maio/2020). Fonte: Dos autores. 
$\mathrm{Na}$ Figura 6, apresentamos algumas estimativas para o Estado do Espírito Santo/ES, com os dados fornecidos pelo Ministério da Saúde, na primeira quinzena de Maio/2020.

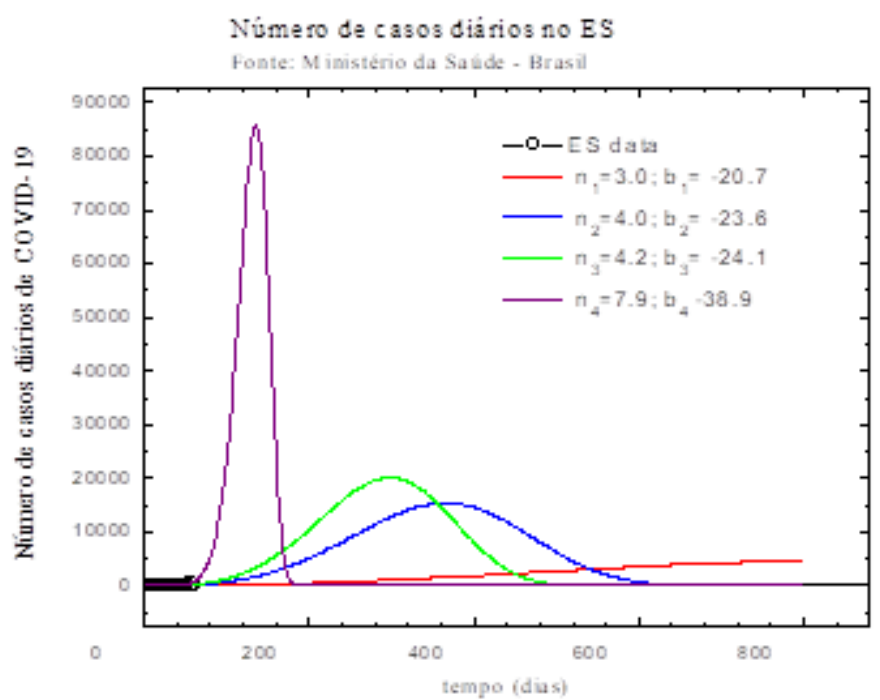

Figura 6. Estimativas realizadas por meio da equação de Avrami (JMAK), para o Estado do Espírito Santo/ES (realizado na primeira quinzena de maio/2020). Fonte: Dos autores.

Na Figura 7, apresentamos algumas estimativas para o Estado do Pará, por meio do modelo preditivo baseado na equação de Avrami:

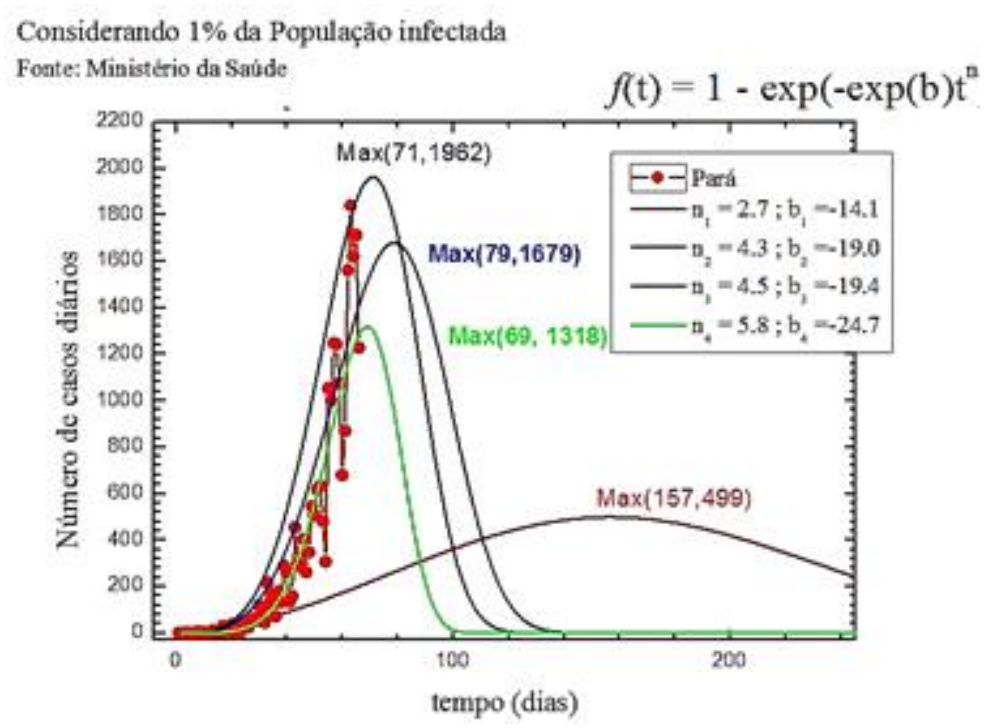

Figura 7. Estimativas realizadas por meio da equação de Avrami (JMAK), para o Estado do Pará/PA (realizado na primeira quinzena de junho/2020). Fonte: Dos autores. 
$\mathrm{Na}$ Figura 8, utilizamos modelos preditivos baseados em Gaussianas, para o Estado do Pará:

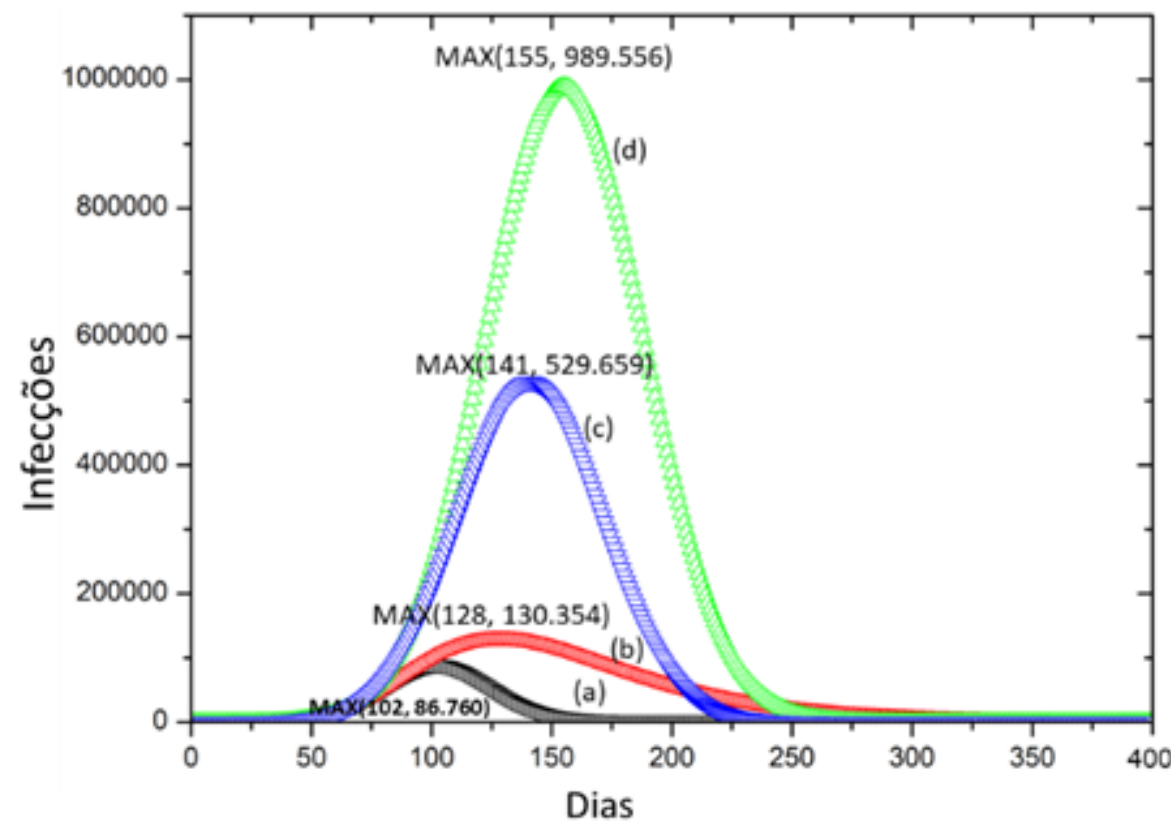

Figura 8: (a) Ajuste não-linear realizado para a curva $y=y_{0}+\left(A /\left(w^{*} \operatorname{sqrt}(P I / 2)\right)\right)^{*} \exp \left(-2^{*}\left(\left(x-x_{c}\right) / w\right)^{\wedge} 2: y_{0}=\right.$ $78,68902, x_{c}=102,45252, w=44,59459, A=4,84572 E 6$, com o ajuste $R^{2}=0,99901$;

(b) Ajuste não-linear realizado para a curva $y=y_{0}+A^{*} \exp (-\exp (-z)-z+1), z=\left(x-x_{c}\right) / w: y_{0}=204,64804, x_{c}=$ $128,47821, w=43,76523, A=130157,7524$, com o ajuste $R^{2}=0,99892$;

(c) Ajuste não-linear realizado para a curva $y=\exp \left(a+b^{*} x+c^{*} x^{\wedge} 2\right)$ : $a=2,28633, x_{c}=140,9635060, b=$ $0,15456, c=-5,48227 \mathrm{E}(-4)$, com o ajuste $R^{2}=0,99933$;

(d) Ajuste não-linear realizado para a curva $y=y_{0}+\left(A /\left(w^{*} \operatorname{sqrt}(P I / 2)\right)\right)^{*} \exp \left(-2^{*}((x-x c) / w)^{\wedge} 2: y_{0}=-61,73036, x_{c}\right.$ $=155,12796, \mathrm{w}=65,52222, \mathrm{~A}=8,1268 \mathrm{E} 6$, com o ajuste $\mathrm{R}^{2}=0,99935$;

Fonte: Adaptado de Nascimento (2020).

Ressaltamos que, em relação à Figura 8 (cujos cálculos foram realizados na semana final de Junho/2020), atualmente os dados oficiais para o Estado do Pará, ainda se encontram entre as curvas (b) e (c), apenas em estimativa de quantitativo de infectados (grifos nossos).

Nessa semana, em que os ajustes não-lineares para o Estado do Pará foram realizados, os números oficiais apontaram para $100 \mathrm{mil}$ infectados. Baseado nas análises realizadas, a curva (d), corresponde à estimativa de números reais de infectados no Pará, significando dizer que: pela falta de ampla testagem realizada na população, em que o número de 100 mil infectados foram os que de fato estiveram diagnosticados por meio de exames, a estimativa em números reais correspondia em $\underline{989.556 \text { (aproximadamente } 1}$ 
milhão) ${ }^{1}$ Paraenses que já tinham tido contado com o Coronavírus ou estavam infectados (Grifos nossos).

Na Figura 8, apresentamos os novos casos de infecções diários e na Figura 9, 0 total de óbitos por meio do modelo preditivo utilizando o modelo SEIAHR (ROCHA FILHO et al., 2020) para o Estado do Pará. As variáveis necessárias ao modelo à resolução das equações diferenciais $\psi, L^{(0)}, \theta, \sigma, \gamma, \zeta, \tau_{1}, \tau_{2}, \chi \mathrm{e} \xi$, apresentam seus valores disponibilizados em ALMEIDA et al. (2020), bem como os demais detalhamentos para as predições:

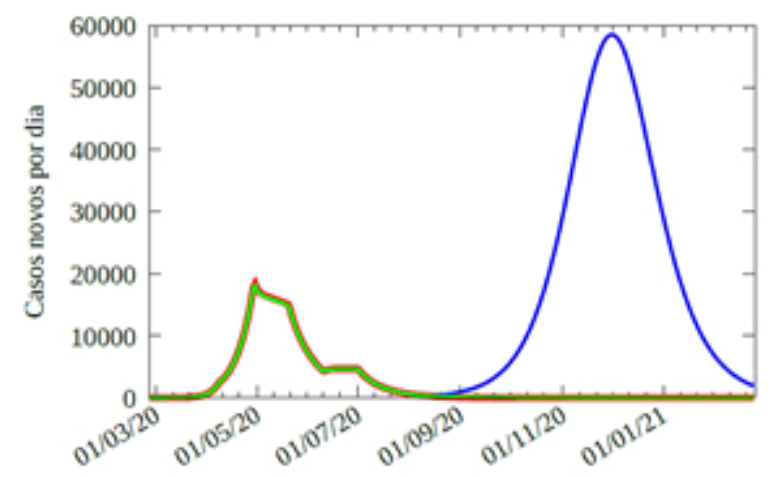

Figura 9. Estimativas realizadas por meio o modelo SEIAHR (ROCHA FILHO et al., 2020; ALMEIDA et al., 2020), para o Estado do Pará/PA (realizado ao fim da primeira quinzena de agosto/2020). Fonte: adaptado de (ALMEIDA et al., 2020).

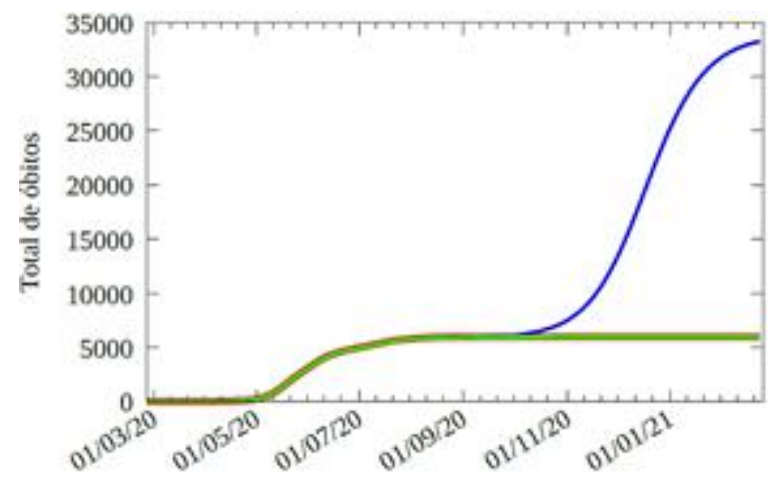

Figura 10. Estimativas realizadas por meio o modelo SEIAHR (ROCHA FILHO et al., 2020; ALMEIDA et al., 2020), para o Estado do Pará/PA (realizado ao fim da primeira quinzena de agosto/2020). Fonte: adaptado de (ALMEIDA et al., 2020).

Pelos resultados apresentados alicerçados no modelo predito SEIAHR, podemos perceber a ratificação das estimativas que realizamos já no começo de $2^{\circ}$ semestre de 2020 para a $2^{a}$ onda de novos casos e óbitos de Covid-19. Como ocorreu de fato, para o final do ano de 2020 e começo do ano de 2021, após o período eleitoral e as festas (Círio 
de Nazaré, natal e réveillon) para o Pará, não foram bons, diante da flexibilização que ocorreu no Estado.

As estimativas, ratificaram também que, a referida flexibilização com a falta de consciência das pessoas, em termos de relaxarem ou até mesmo abandonarem as medidas de profilaxia recomendadas para Organização Mundial de Saúde (OMS) e até mesmo o Ministério da Saúde, consequentemente, realizaram incomensuráveis aglomerações, resultado na já sobrecarga na rede privada de saúde e elevado o aumento na procura por atendimento médico na rede pública de saúde, por casos de Covid-19.

Semelhantemente às Figura 9 e Figura 10, apresentamos nas Figura 10 e Figura 11, os novos casos de infecções diários para o Estado do Espírito Santo/ES e o total de óbitos, respectivamente, utilizando o modelo SEIAHR. Seguindo ainda de forma atenda as observações citadas anteriormente em relação às equações diferenciais, seguem os gráficos com informações estimativas:

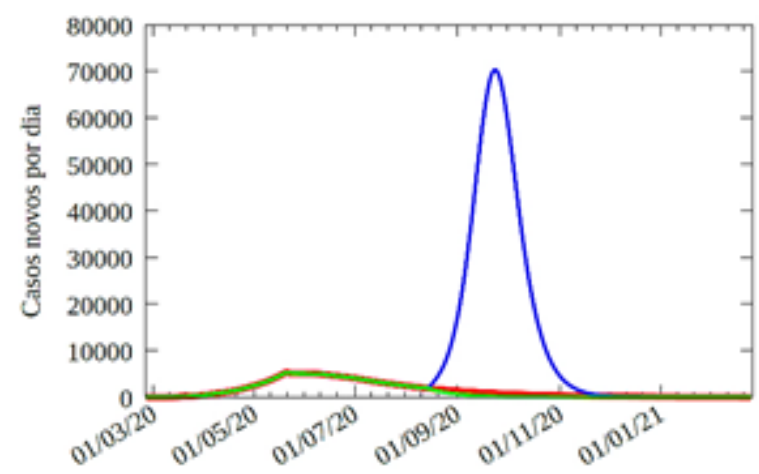

Figura 11. Estimativas realizadas por meio o modelo SEIAHR (ROCHA FILHO et al., 2020; ALMEIDA et al., 2020), para o Estado do Espírito Santo, para as novas infecções diárias (realizado ao fim da primeira quinzena de agosto/2020). Fonte: adaptado de ALMEIDA et al., 2020.

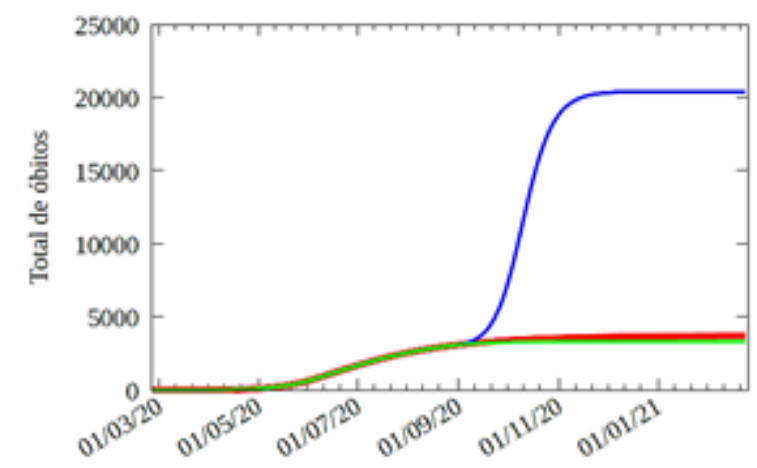

Figura 12. Estimativas realizadas por meio o modelo SEIAHR (ROCHA FILHO et al., 2020; ALMEIDA et al., 2020), para o Estado do Espírito Santo, considerando o total de óbitos (realizado ao fim da primeira quinzena de agosto/2020). Fonte: adaptado de ALMEIDA et al., 2020. 


\subsection{Medidas de correlações entre os Estados}

Por fim, realizamos as medidas de correlações especificamente para os estados do Espírito Santo e Pará. Desta forma, por meio das séries temporais representativas de cada Estado Brasileiro e o DF, a verificação de que, em qual nível há correlação entre PA e os 25 Estados e o DF, assim como o ES os 25 Estados e o DF, foi possível. Ratificamos que nesta pesquisa, objetivamos analisar os números de novas infecções e óbitos diários, conforme os dados oficiais disponibilizados pelo Ministério da Saúde.

Para cada par de Estados analisados, um correspondente coeficiente de correlação individualizado foi encontrado. A título de exemplo, Espírito Santo e Paraíba (ES x PB), o coeficiente de correlação de Spearman, correspondeu a $r_{s}=0,75732$. Em relação ao número de novas infecções diárias, para o Estado do Espírito Santo temos os seguintes resultados, conforme Tabela 1:

Tabela 1. Correlação medida entre o Estado do Espírito Santo com os demais Estados e o DF. Fonte: Dos autores.

\begin{tabular}{c|c|c|c|c|c|c}
\hline \multicolumn{1}{c}{$\begin{array}{c}\text { Correlações entre o estado do ES } \\
\text { com os demais Estados e DF) }\end{array}$} & \multicolumn{2}{c|}{$r$} & \multicolumn{2}{|c}{$r_{S}$} & \multicolumn{3}{c}{$\tau$} \\
\hline ES & DF & 0,66839 & PB & 0,7734 & AL & 0,60342 \\
\hline ES & PI & 0,64897 & MA & 0,76653 & CE & 0,60121 \\
\hline ES & PB & 0,64524 & AL & 0,75732 & To & 0,59521 \\
\hline ES & RO & 0,61733 & RN & 0,7567 & PE & 0,5876 \\
\hline ES & BA & 0,61455 & SC & 0,75103 & PR & 0,58105 \\
\hline ES & PA & 0,58824 & AP & 0,74775 & SE & 0,57626 \\
\hline ES & MT & 0,57742 & MG & 0,74355 & MT & 0,57012 \\
\hline ES & MG & 0,57234 & SE & 0,7236 & SP & 0,55293 \\
\hline ES & AC & 0,56106 & PA & 0,7157 & AP & 0,54794 \\
\hline ES & SP & 0,55804 & RS & 0,70327 & AC & 0,53727 \\
\hline ES & AL & 0,55341 & TO & 0,69304 & RS & 0,52582 \\
\hline ES & PR & 0,52874 & PI & 0,68722 & MA & 0,52471 \\
\hline ES & RR & 0,52724 & GO & 0,68637 & MS & 0,52185 \\
\hline ES & SE & 0,51838 & SP & 0,68623 & RJ & 0,51602 \\
\hline ES & MA & 0,51598 & CE & 0,68348 & AM & 0,50933 \\
\hline ES & MS & 0,5041 & AM & 0,68299 & BA & 0,50868 \\
\hline ES & AM & 0,49408 & MS & 0,67379 & RR & 0,5079 \\
\hline & & & & &
\end{tabular}




\begin{tabular}{c|c|c|c|c|c|c}
\hline ES & RJ & 0,4922 & RO & 0,66549 & DF & 0,5054 \\
\hline ES & PE & 0,47077 & RR & 0,66539 & PA & 0,50351 \\
\hline ES & RS & 0,45569 & AC & 0,66264 & GO & 0,50309 \\
\hline ES & RN & 0,44819 & PE & 0,661 & RN & 0,49716 \\
\hline ES & GO & 0,44636 & DF & 0,64972 & MG & 0,49084 \\
\hline ES & TO & 0,43174 & RJ & 0,6423 & RO & 0,47617 \\
\hline ES & AP & 0,40757 & MT & 0,64037 & PARAÍBA & 0,47404 \\
\hline ES & CE & 0,40472 & BA & 0,59346 & SC & 0,44255 \\
\hline ES & SC & 0,36662 & PR & 0,56263 & PI & 0,41191 \\
\hline
\end{tabular}

Ratificando o que dissemos anteriormente em relação ao coeficiente de Pearson: quando $r=+1$ a correlação é perfeitamente positiva. Se $r=-1$ a correlação é perfeitamente negativa e se $r=0$ não há correlação entre os pontos ou há, porém, é nãolinear [1-12]. Conforme Mukaka [17], para os coeficientes de Pearson e Spearman as correlações fortes são aquelas em que os valores estão compreendidos entre 0,71 e 0,9 e/ou -0,71 e -0,9. Já as correlações muito fortes, correspondem para os valores maiores que 0,9 ou menores que -0,9) [17]. Desta forma, apresentaremos nas Tabela 1 e Tabela 2, índices de correlações presentes na pesquisa, para o Espírito Santo. Pelo exporto em Mukaka [17], adotamos o coeficiente de correlação para postos de Spearman $\left(r_{s}\right)$, para valores a partir de 0,7, como ponto de corte na apresentação das informações nas tabelas a seguir. Apresentamos na Tabela 1. Em relação ao número de óbitos diários, para o Estado do Espírito Santo temos os seguintes resultados, Tabela 2:

Tabela 2. Coeficiente de correlação de Pearson $(r)$, Spearman $\left(r_{s}\right)$ e Kendall $(\tau)$, calculados entre ES e os demais Estados (e o DF), para o número de óbitos diários de Covid-19. Fonte: Dos autores.

\begin{tabular}{l|l|l|l|l|l|l}
\hline \multicolumn{2}{c|}{$r$} & \multicolumn{2}{c|}{$r_{S}$} & \multicolumn{2}{c}{$\tau$} \\
\hline ES & AL & 0,78618 & AL & 0,81511 & AL & 0,65831 \\
\hline ES & MA & 0,77499 & SE & 0,80885 & SE & 0,63835 \\
\hline ES & SE & 0,71441 & MA & 0,79941 & MA & 0,63737 \\
\hline ES & PB & 0,68131 & PB & 0,77972 & PB & 0,61171 \\
\hline ES & PI & 0,66458 & RO & 0,75062 & RO & 0,58987 \\
\hline ES & RO & 0,66019 & PI & 0,7264 & RN & 0,56116 \\
\hline ES & AC & 0,63276 & RN & 0,71659 & PI & 0,5526 \\
\hline
\end{tabular}


Em relação ao número de novas infecções diárias, para o Estado do Pará temos os seguintes resultados, conforme Tabela 3:

Tabela 3. Coeficiente de correlação de Pearson $(r)$, Spearman $\left(r_{s}\right)$ e Kendall $(\tau)$, calculados entre PA e os demais Estados (e o DF), para o número de novos casos diários de Covid-19. Fonte: Dos autores.

\begin{tabular}{|c|c|c|c|c|c|c|}
\hline $\begin{array}{c}\text { Correlações entre o } \\
\text { estado do PA com os } \\
\text { demais Estados e o } \\
\text { DF) }\end{array}$ & & $r$ & & $r_{S}$ & & $\tau$ \\
\hline$P A$ & $\mathrm{MA}$ & 0,84203 & $\mathrm{DF}$ & 0,90119 & PB & 0,74368 \\
\hline PA & PB & 0,83719 & $\mathrm{RO}$ & 0,90016 & CE & 0,7369 \\
\hline PA & AM & 0,73845 & $A C$ & 0,81825 & AM & \begin{tabular}{|l|}
0,65114 \\
\end{tabular} \\
\hline PA & $\overline{\mathrm{AL}}$ & 0,7261 & RJ & 0,81711 & $\mathrm{AL}$ & 0,63747 \\
\hline PA & $A C$ & 0,70731 & $\mathrm{AL}$ & 0,79959 & RJ & 0,62456 \\
\hline PA & PE & 0,67035 & ES & 0,79703 & PE & 0,61975 \\
\hline $\mathrm{PA}$ & RO & 0,64366 & CE & 0,79405 & AP & 0,61908 \\
\hline PA & $\mathrm{BA}$ & 0,64348 & $\mathrm{PI}$ & 0,78437 & SP & 0,61614 \\
\hline PA & $\mathrm{PI}$ & 0,63416 & $\mathrm{PE}$ & 0,78126 & SC & \begin{tabular}{|l|l|}
0,61259 \\
\end{tabular} \\
\hline PA & SP & 0,63337 & RR & 0,7808 & $\mathrm{RO}$ & 0,60975 \\
\hline $\mathrm{PA}$ & DF & 0,61662 & PB & 0,77999 & ES & 0,60903 \\
\hline PA & ES & 0,58824 & AP & 0,77637 & $\mathrm{PI}$ & 0,60601 \\
\hline PA & RJ & 0,57525 & AM & 0,77417 & $A C$ & 0,60101 \\
\hline PA & $\mathrm{AP}$ & 0,55841 & BA & 0,76711 & BA & 0,59602 \\
\hline PA & $\mathrm{RR}$ & 0,55298 & SC & 0,73391 & MS & 0,5596 \\
\hline $\mathrm{PA}$ & SE & 0,53238 & MA & 0,72384 & DF & 0,54174 \\
\hline PA & TO & 0,49225 & MG & 0,70244 & To & 0,52964 \\
\hline PA & $M G$ & 0,48354 & TO & 0,68299 & $\mathrm{RR}$ & 0,52399 \\
\hline PA & CE & 0,47007 & SP & 0,67271 & MG & 0,52185 \\
\hline PA & MT & 0,44184 & SE & 0,66535 & MA & \begin{tabular}{|l|}
0,50575 \\
\end{tabular} \\
\hline PA & MS & 0,39914 & $\mathrm{RN}$ & 0,64733 & $\mathrm{GO}$ & 0,48398 \\
\hline PA & GO & 0,37145 & PR & 0,62843 & $\mathrm{RS}$ & 0,46166 \\
\hline PA & $\mathrm{RS}$ & 0,31589 & $\mathrm{RS}$ & 0,62669 & MT & 0,4587 \\
\hline PA & $\mathrm{RN}$ & 0,31366 & $\mathrm{GO}$ & 0,59354 & PR & 0,42135 \\
\hline PA & PR & 0,26461 & MT & 0,57591 & SE & 0,42083 \\
\hline PA & SC & 0,12211 & MS & 0,56442 & $\mathrm{RN}$ & 0,40689 \\
\hline
\end{tabular}

Tabela 4. Coeficiente de correlação de Pearson $(r)$, Spearman $\left(r_{s}\right)$ e Kendall $(\tau)$, calculados entre PA e os demais Estados (e o DF), para o número de óbitos diários de Covid-19. Fonte: Dos autores.

\begin{tabular}{c|c|c|c|c|c|c}
\hline \multicolumn{3}{c|}{$r$} & \multicolumn{2}{c|}{$r_{S}$} & \multicolumn{2}{c}{$\tau$} \\
\hline PA & CE & 0,71093 & MA & 0,84872 & MA & 0,69022 \\
\hline PA & PE & 0,69166 & PE & 0,8279 & PE & 0,66429 \\
\hline PA & MA & 0,60904 & AL & 0,79099 & AL & 0,63251 \\
\hline PA & AP & 0,59473 & CE & 0,75782 & CE & 0,5938 \\
\hline
\end{tabular}




\begin{tabular}{l|l|l|l|l|l|l}
\hline PA & RJ & 0,58276 & AP & 0,72579 & AP & 0,57355 \\
\hline
\end{tabular}

Por meio das séries temporais construídas especificamente dos coeficientes aqui utilizados, Pearson $(r)$, Spearman $\left(r_{s}\right)$, e Kendall $(\tau)$, foi possível visualizarmos na Figura 13 e Figura 14 as 3 métricas de correlações apresentadas nesta pesquisa. Destacamos que os índices "i" e "o" contidos nos gráficos, representam respectivamente "número de infectados diários" e "número de óbitos diários".

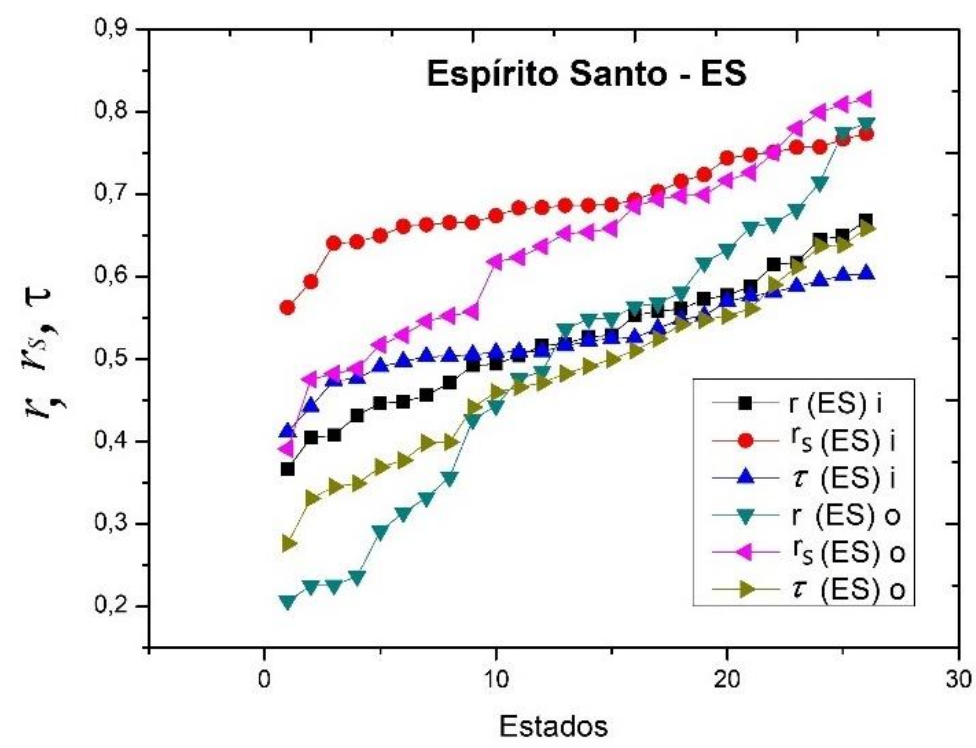

Figura 13. Padrão evolutivo dos coeficientes de correlações, para o Estado do Espírito Santo. Fonte: Dos autores.

De forma análoga à figura anterior, para o Estado do Pará, apresentamos a Figura 11:

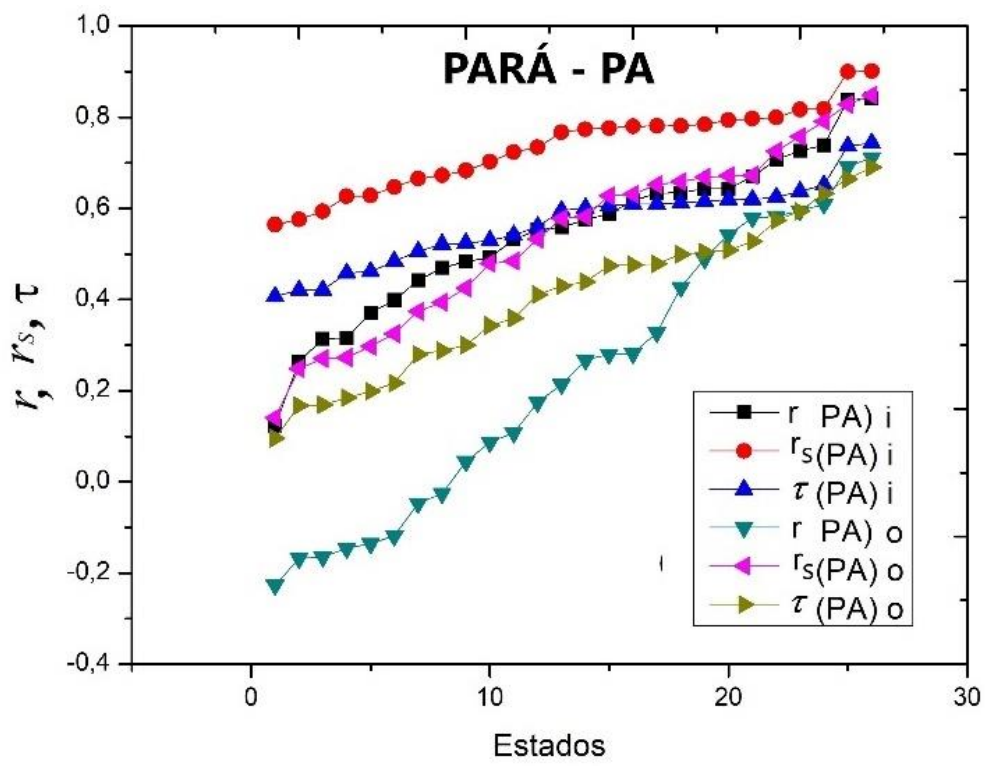


Figura 14. Padrão evolutivo dos coeficientes de correlações, para o Estado do Pará. Fonte: Dos autores.

Tanto pela Figura 13 quanto a Figura 14, podemos observar que, as séries temporais construídas observando a correlação entre os X e Y Estados Brasileiros, cada ponto, corresponde a um par, quer seja para o Para x (Estado Brasileiro e o DF), quer seja o Espírito Santo x (Estado Brasileiro e o DF). Com as análises destas figuras, podemos observar do menor valor de correlação entre os pares de Estados até o maior. Desta forma, diante deste cenário epidemiológico entrados para o período de análise (dezembro/2020), temos neste artigo, como avaliar em que medida ocorreu a difusão do Coronavírus nos diversos Estados, pela comparação nos padrões singulares a cada local.

Diante das análises realizadas utilizando os diferentes coeficientes de correlação para analisar especificamente os Estados do Pará e do Espírito com os demais Estados Brasileiros e do DF. O coeficiente de correlação de Spearman $\left(r_{s}\right)$, apresentou-se em destaque nas medidas realizadas. Como era de se esperar, o padrão de comportamento do coeficiente de correlação por postos de Kendall $(\tau)$, apresentou-se "idêntico" ao coeficiente de correlação por postos de Spearman $\left(r_{s}\right)$.

De uma forma geral, podemos afirmar para o Espírito Santo, em termos do número diário de infecções que, que ele está, está fortemente correlacionado com os Estados do Norte e Nordeste e sutilmente com o Sudeste e Centro-Oeste. Em relação às correlações específicas (correlação forte) para o número de óbitos, há uma predominância do ES com a região Nordeste e com a região Norte, respectivamente. Semelhantemente, o Estado do Pará apresentou uma correlação fortíssima com a região Nordeste, em relação ao número diário de infectados, em que destacamos os Estados da Paraíba e Maranhão. O padrão de uma forte correlação, conforme Mukaka (2012), também ocorreu com a própria região Norte e, também, Sudeste e Centro-Oeste. Para as correlações do Estado do Pará em relação ao número de óbitos diários, houve uma predominância com a região Nordeste e com a região Norte, respectivamente.

\section{CONSIDERAÇOES FINAIS}

Como dito durante este artigo, nosso objetivo aqui foi o de apresentar cenários epidemiológicos à difusão do Coronavírus. Indicamos/sugerimos 5 metodologias diferentes para que pudéssemos realizar estimativas em relação à difusão do SARS-CoV2. Longe de nossas pretensões limitarmos o quantitativo de modelos de previsão à 
utilização em estimativas epidêmicas. Contudo, dos diversos modelos epidemiológicos presentes na literatura científica, ratificamos que por uma questão de escolha dos autores, apresentamos aqui o modelo SIR, o modelo SEIRD, o modelo de Física do Estado Sólido (Avrami ou JMAK), o modelo SEIAHR e os ajuste baseados em Gaussianas.

Dos métodos que apresentamos, escolhemos também para apresentação dos resultados: a equação de Avrami ou JMAK, as Gaussianas e o modelo SEIAHR. Com os resultados parciais apresentados aqui, apresentamos a necessidade de realizarmos análises dos dados confiáveis para dar suporte as políticas públicas eficientes para o combate à Pandemia. Desta forma, nossa expectativa foi de forma suscinta, colaborar com a compreensão do quadro epidemiológico por meio de cenários epidemiológicos testados por modelos matemáticos que descrevem a difusão da doença infecciosa de transmissão pessoa a pessoa. E predizer quais parâmetros variam em cada cenário.

Em relação às medidas de correlações, ao tratarmos os Estados Brasileiros e o DF como séries temporais, foi possível utilizarmos os coeficientes de correlações trabalhados neste artigo, bom uma base estatística e matemática, realizando análises físicas pela observância da natureza, um fenômeno não-linear: uma pandemia.

Não foi nosso foco detalhar as nuances estatísticas aqui apresentadas, porém, demonstramos de forma didática como trabalhar (por meio deste artigo), realizando nossa proposição de análise à difusão do Coronavírus, observando as métricas de correlação. Desta forma, pudemos perceber que tanto o Estado do Espírito Santo quanto o Pará, estão fortemente correlacionados com as regiões Norte e Nordestes e até, com uma fortíssima correlação entre, especificamente o Pará e as supracitadas regiões. Por fim, apresentamos como cada índice estatístico se comportou em seu processo de "evolução", partindo dos menores valores de correlação entre um par de Estados até o maior.

\section{AGRADECIMENTOS}

Jefferson Nascimento agradece a oportunidade de ter realizado pós-doutoramento no departamento de modelagem computacional no CIMATEC/BA (em Sistemas Complexos - Cenários Epidemiológicos, cujo foco foi a difusão do Coronavírus no Brasil). Estéfano Aparecido Vieira agradece ao CNPq por conta de sua bolsa de Produtividade Desen. Tec. e Extensão Inovadora. Marcelo A. Moret agradece ao CNPq pelo suporte 
financeiro parcial oriundo de sua bolsa de Produtividade em Pesquisa (No. 305291/20181).

\section{REFERÉNCIAS}

ALMEIDA, A.C.G.; CORDEIRO, A. J. A.; SCORZA, F. A.; MORET, M. A.; ROCHA FILHO, T. M.; RAMALHO, W. M. Nota Técnica (13/08/2020) - Situação da pandemia de Covid19 no Brasil. UNB, Brasília, DF, 2020.

ANG, A.H.S., \& TANG, W.H. Probability Concepts in Engineering, Planning and Design, Vol. II, John Willey and Sons, New York, USA, 1984.

BAUER, Lidiane. Estimação do coeficiente de correlação de spearman ponderado. 2007. 95 f. Dissertação (Mestrado em Epidemiologia). Curso de PósGraduação em Epidemiologia, Faculdade de Medicina, Universidade Federal do Rio Grande do Sul, Porto Alegre, 2007.

BRITO, A. C.; VINHAS, G. M.; ALMEIDA, Y. M. B. de, Wellen, M. R. W.; Canedo, E. L.; Rabello, M. S. Efeito da adição de PHB na cristalização a frio do PET. Polímeros, 22(2), 111-116. doi.org/10.1590/S0104-14282012005000016, 2012.

CINTRA, H. P. C; FONTINELE, F. N. Estimative of real number of infections by COVID-19 on Brazil and possible scenarios. The preprint server for Health Sciences, 2020 (Preprint). Disponível em <https://doi.org/10.1101/2020.05.03.20052779>. Acesso em: 11 Nov. 2020.

CONOVER, W. J. Practical nonparametric statistics. 3rd . ed. New York: John Wiley \& Sons, 1998.

IBARRA, M. A. C. Análise comparativa de métodos para estimativa de valores extremos de processos aleatórios não-gaussianos. 106f. Dissertação (Mestrado Programa de Engenharia Civil) - UFRJ / COPPE /, 2017.

KERMACK, W. O.; MCKENDRICK, A. G. Contributions to the mathematical theory of epidemics I-1927. Bull. Math. Biol. 53, 33-55, 1991.

MIOT, H. Análise de correlação em estudos clínicos e experimentais. Jornal Vascular Brasileiro, 17, 275-279, 2018.

MUKAKA, M. M. Statistics corner: A guide to appropriate use of correlation coefficient in medical research. Malawi Med J. 24(3):69-71. PMid:23638278, 2012.

NASCIMENTO, J. O. do. Redes sociais e complexas: redes semânticas do Ensino de Física Brasileiro. 123f. Tese (Doutorado em modelagem computacional e tecnologia industrial) - CIMATEC, Salvador, 28 jun. 2019. 
NASCIMENTO, J. O. do. Relatório técnico de atividades de pós-doutorado. Salvador/BA: Sistemas Complexos - Cenários Epidemiológicos, 2020. Relatório (Pósdoutorado) - Departamento/Programa de Modelagem Computacional, CIMATEC, 2020.

PASSOS, C. A. C.; VIEIRA, E. A.; LOURENCO, J. A.; NASCIMENTO, J. O. do. Characteristics and evolution of COVID-19 cases in Brazil: mathematical modeling and simulation. medRxiv - the preprint server for Health Sciences (Preprint). Disponível em < https://www.medrxiv.org/content/10.1101/2020.10.14.20212829v1>. Acesso em: 09 Nov. 2020. doi: https://doi.org/10.1101/2020.10.14.20212829, 2020.

ROCHA FILHO, T. M.; SANTOS, F. S. G. dos; GOMES, V. B.; ROCHA, T. A. H.; CRODA, J. H. R.; RAMALHO, W. M.; ARAÚJO, W. N. de. Expected impact of COVID-19 outbreak in a major metropolitan area in Brazil. The preprint server for Health Sciences, 2020. (Preprint). Disponível em https://www.medrxiv.org/content/10.1101/2020.10.14.20212829v1>. Acesso em: 12 Nov. 2020. doi: https://doi.org/10.1101/2020.10.14.20212829.

SANTOS, S. S. Estudo comparativo de medidas de dependência e aplicações em dados de expressão gênica. Universidade de São Paulo, USP, São Paulo, 2012.

SAVI, P V.; SAVI, M. A.; BORGES, B. A mathematical description of the dynamics of coronavirus disease 2019 (COVID-19): A case study of Brazil. (Preprint). Disponível em <http://arxiv.org/abs/2004.03495>. Acesso em: 15 Nov. 2020.

SHI, J.; HE, F.; XIE, J., LIU, X.; YANG, H. Kinetic analysis of crystallization in Li2O-Al2O3SiO2-B2O3-BaO glass-ceramics, Journal of Non-Crystalline Solids, Volume 491, Pages 106-113, ISSN 0022-3093, doi.org/10.1016/j.jnoncrysol.2018.04.004, 2018 